social role of masculinity may explain the high effect magnitude in men. Given that women are more frequent targets of sexual harassment, a sexist social and organisational context may mask the effect of this unacceptable violence in women. Also, there might be a possible normalization of the practice with necessary adaptation of women.

\section{0-486 GENDER-BASED VIOLENCE AND HARASSMENT AND THE INCIDENT PURCHASE OF PSYCHOTROPIC MEDICATION. A PROSPECTIVE COHORT STUDY ON THE SWEDISH WORKING POPULATION}

${ }^{1}$ Katrina Blindow, Johan Paulin, Kristina Johnell, Anna Nyberg. 'Karolinska Institutet, Sweden

\subsection{6/OEM-2021-EPI.138}

Introduction Gender-based violence and harassment (GBVH) in the workplace has been found to be associated with selfreported mental ill-health. The prospective risk of using psychotropic medication for the treatment of common mental disorders (CMD) is unclear, though.

Objectives To estimate the prospective association between the exposure to three types of GBVH and the purchase of psychotropic medication in a large population-based Swedish study.

Methods Survey data from the biannual Swedish Work Environment Survey (SWES) from 2007 to 2013 (N=23 452) were merged with data on antidepressants, sedatives/hypnotics and anxiolytics from the Swedish Prescribed Drug Register. Exposure to GBVH was measured as sexual harassment 1) from workplace personnel, 2) from non-workplace personnel (e.g., customers or clients), and 3) gender harassment from workplace personnel. Gender-stratified cox proportional hazards analyses with days to outtake as the time-scale and first instance of medicine outtake as the failure event were fitted for each exposure. Analyses were adjusted for age, parental migration background, education, income, family situation, and labor market industry.

Results In men, weekly to daily exposure to gender harassment (HR 2.39, 95\% CI 1.35 - 4.23) and in women, gender harassment once in 12 months (HR 1.18, 95\% CI 1.02 1.36) and weekly to daily (HR $1.62,95 \%$ CI 1.13 - 2.31) were associated with prospective medication outtake. In women, exposure to sexual harassment monthly from workplace personnel (HR 1.60, 95\% CI 1.03 - 2.49) and from non-workplace personnel (HR 1.34, 95\% CI 1.00 - 1.79) were also associated with medication purchase.

Conclusion Exposure to gender harassment at the workplace may contribute to the development of CMD, particularly when it occurs more frequently. Sexual harassment seems to impact the mental health of women, regardless if it stems from individuals inside or outside the organization.

\section{Shiftwork}

\section{$0-47$ \\ BEYOND NIGHT WORK: WHY SHIFT WORK WITHOUT NIGHT WORK MAY STILL BE HARMFUL}

${ }^{1}$ Jacqueline Ferguson, Aki Koskinen, Mikko Härmä. 'Stanford University, United States
Objective Most literature examining the health effects of shift work prioritize night work as the exposure of interest. However, little attention has been paid to the co-occurrence of working time characteristics beyond shift type (e.g. night vs day) that may lead to circadian rhythm disruption; characteristics such as shift intensity, shift duration, rotation pattern, and weekend work. We hypothesize that the co-occurrence of these characteristics with and without night work could explain why shift work without night work is sometimes associated with adverse health effects.

Methods Time-registry data on 14,430 full-time (>150 shifts/ year) healthcare workers from 2012-2016 were sourced from the Working Hours in the Finnish Public Sector (WHFPS) study to describe the prevalence and co-occurrence of working time characteristics that may lead to circadian rhythm disruption. First, each characteristic (type, intensity, duration, rotational pattern, and weekend work) was cross-classified in a matrix to examine its co-occurrence with all other characteristics (e.g. how many night shifts were also long shifts). Second, the prevalence of each working hour characteristic by annual shift schedules (permanent or rotating day/evening/night) were examined.

Results Our results provide evidence that working hour characteristics hypothesized to cause circadian rhythm disruption have a varying distribution with each other and across shift schedules- even schedules that don't include nights. While day shifts are thought to not cause circadian rhythm disruption, $32 \%$ of day shifts versus $34 \%$ of night shifts cooccurred with long work hours, quick returns, and rotations. Furthermore, despite not including nights, the Day/Evening schedule had more quick returns than the Day/Evening/Night schedule and still contained rotations, long hours, and weekend work.

Conclusion Thus, a cautious interpretation of the association between night work and human health may be warranted, as circadian rhythm disruption may be caused by long hours, rotations, or quick returns which may or may not accompany night work.

\section{0-148 SHIFT WORK AND BREAST CANCER. A COHORT STUDY FROM FINLAND BASED ON SURVEY AND PAYROLL DATA}

${ }^{1}$ Mikko Härmä, Anneli Ojajärvi, Aki Koskinen, Jenny-Anne Lie, Mika Kivimäki, Johnni Hansen. ${ }^{1}$ Finnish Institute of Occupational Health, Finland

\subsection{6/OEM-2021-EPI.140}

Introduction International Agency for Research on Cancer (IARC) has concluded that night shift work is probably carcinogenic to humans.

Objectives To examine the association of shift work with breast cancer among women in the Finnish public sector.

Methods We investigated the associations of night and shift work, and potential confounders, with incident breast cancer using a survey $(\mathrm{N}=64$ 082, mean age 42,3 years) and payroll cohort ( $\mathrm{N}=17$ 286, 2008-) of the Finnish Public Sector study (FPS). Survey and payroll employees (baseline 2000-2012) were linked to National Cancer Register and followed-up for breast cancer to the end of 2016. Hazard ratios (HR) and confidence intervals (95\% CI) from Cox proportional hazard regression models were calculated, including adjustment for 
age, SES, number of children, smoking, alcohol and body mass index.

Results 2057 incident cases of breast cancer were identified. Compared to day work, self-reported shift work without nights at baseline was associated with an increased risk of breast cancer during a follow-up period of less than 10 year, after adjustment (HR 1.33, 95\% CI 1.15-1.55). In contrast, follow-up for $>=10$ years showed no increased HR. Night shift work was most notably (HR 2.05, 95\% 1.04-4.01) associated with breast cancer among employees 50 years or older after 10 years of follow-up. The risk of breast cancer tended to depend on earlier exposure time. The results of the more recent payroll sub-cohort showed no association of shift work and breast cancer, including intensity and duration of night shift work.

Conclusion This study gives some indications of an increased risk of breast cancer among subgroups of shift-working Finnish public sector employees. However, insufficient information on past exposure to, and intensity of night work, limits the ability to draw firm conclusions.

\section{0-225 EXPLORING THE IMPACT OF NIGHT SHIFT WORK AND MELATONIN ON METHYLATION IN CIRCADIAN GENES}

'Jennifer Ritonja, Parveen Bhatti, Lisa Flaten, Danai G Topouza, Qing Ling Duan, Michael Leung, Francine Durocher, Joan E Tranmer, Kristan J Aronson. 'Queen's University, Canada

\subsection{6/OEM-2021-EPI.141}

Introduction Night shift work is associated with increased cancer risk, but the molecular mechanisms are not well-understood. It is hypothesized that melatonin suppression due to night shift work could impact DNA methylation in circadian genes, although this has been evaluated by few studies.

Objectives This study explored the relationship between night shift work parameters and patterns of melatonin secretion on methylation in circadian genes among women.

Methods A cross-sectional study was conducted in 2019-2020 among 74 female healthcare employees who participated in a previous study in which urinary melatonin levels were evaluated over a 48-hour period. Participants provided information on demographics, lifestyle behaviors, and night shift work such as current night shift work pattern, duration in years, and intensity (consecutive nights). The Illumina Infinium MethylationEPIC beadchip was applied to DNA extracted from new blood samples to measure methylation at 1150 CpG loci across 22 circadian genes. Multiple linear regression was used to examine the association between night shift work, melatonin parameters and methylation levels at each $\mathrm{CpG}$ site, while accounting for the false-discovery rate $(q=0.2)$.

Results Compared to day workers, night shift workers had hypermethylation in the promoter region of CSNK1E $(q=0.15)$. Women who worked night shifts for $\geq 10$ years exhibited hypomethylation in the body of NR1D1 $(q=0.08)$ compared to those with $<10$ years of history. Hypermethylation in the body of ARNTL was also apparent for those who worked $\geq 3$ consecutive night shifts a week compared to $<3$ nights $(\mathrm{q}=0.18)$. Among night shift workers, melatonin patterns (24-hr concentrations, peak timing) were associated with methylation at three loci (RORA, MTNR1A, PER3) (q $\leq$ 0.20 ). No association between melatonin and methylation was identified among day workers.
Conclusion These findings suggest that circadian misalignment among night shift workers is associated with differential methylation in several circadian genes, but larger studies are needed to confirm.

\section{0-313 NIGHT SHIFT WORK, SLEEP DISORDERS AND LUNG CANCER RISK AMONG WOMEN: RESULTS FROM A POPULATION-BASED CASE-CONTROL STUDY IN FRANCE (THE WELCA STUDY)}

${ }^{1}$ Emilie Cordina-Duverger, Nastassia Tvardik, Diane Martin, Shreeshti Uchai, Regine Billmann, Pascal Guenel, Loredana Radoï. 'INSERM, France

\subsection{6/OEM-2021-EPI.142}

Introduction Night shift work may lead to sleep disorders and circadian rhythm disruption. It was classified as probably carcinogenic by IARC but, unlike breast or prostate cancer, there is only scant evidence of an association with lung cancer.

Objective To explore the role of night shift work and sleep disorders in lung cancer risk among women.

Methods Information on night work and sleep disorders over lifetime was obtained in a case-control study on female lung cancer in 716 cases and 758 population controls in the Paris area (WELCA). Logistic regression models adjusting for tobacco smoking and other relevant confounders were used to estimate odds ratios (OR) and 95\% confidence intervals (CI) associated with night work exposure metrics (years of night work, frequency of night shifts), sleep duration per day ( $<7 \mathrm{~h}, 7 \mathrm{~h}, \geq 8 \mathrm{~h}$ ) and sleep disorders (difficulty in falling asleep; waking up at night, too early or too tired; intake of sleep medicine). A sleep disruption index (SDI) was used to classify women by number of sleep disturbances in categories of low ( 0 or 1$)$, medium ( 2 or 3 ) or high SDI (4 or 5).

Results The OR was 1.08 (95\% CI 0.75-1.56) in women who ever worked at night. This OR did not increase with duration or frequency of night shifts. Women who slept 8 or more hours per day had an OR of 1.40 (95\% CI 1.04-1.87) as compared to those who slept 7 hours. Women who had both high SDI and who worked at night for 6 or more years had an OR of 3.61 (95\% CI: 1.27-10.23).

Conclusion Lung cancer risk among women was not associated with ever working at night in our study. The association with longer sleep duration is intriguing and warrants further scrutiny.

\section{0-384 THE ASSOCIATION BETWEEN NIGHT SHIFT WORK AND RISK OF BREAST CANCER: RESULTS FROM THE NIGHTINGALE STUDY}

${ }^{1}$ Nina Berentzen, Anouk Pijpe, Roel Vermeulen, Jelle Vlaanderen, Hans Kromhout, Flora van Leeuwen, Matti Rookus. 'Netherlands Cancer Institute, Netherlands

\subsection{6/OEM-2021-EPI.143}

Introduction and Objectives The International Agency for Research on Cancer confirmed night shift work as a 'probable' carcinogen in an updated evaluation (2019). Noting that evidence from human studies is still inconclusive, in particular due to the lack of prospective cohort studies with detailed exposure assessment. We prospectively examined different 